\title{
Promotion to Innovation, Understanding and Transfer of Technology Through Hubs Projects Promoted by the National Secretariat of Higher Education
}

VII International Congress of

Science, Technology,

Entrepreneurship and

Innovation (SECTEI 2020)

Corresponding Author:

R. Villacrés

rvillacres@espoch.edu.ec

Published: 26 August 2021

Production and Hosting by

Knowledge E

(c) R. Villacrés et al. This article is distributed under the terms of the Creative Commons Attribution License, which permits unrestricted use and redistribution provided that the original author and source are credited.

\section{Fomento a la Innovación, Emprendimiento y Transferencia de Tecnología a Través de los Proyectos Hubs Impulsados por la Secretaría Nacional de Educación Superior}

\author{
R. Villacrés, P Arguello, and V. Naranjo
}

Carrera de Administración de Empresas, Facultad de Administración de Empresas, Escuela Superior Politécnica de Chimborazo, Riobamba, Ecuador

\section{Abstract}

This article allows us to expose the promotion of innovation, entrepreneurship and technology transfer and the importance of a society demanding new things, as a result of which there is an increasingly demanding need for satisfaction for those who consume them and make the generation of new products, goods or services are mostly in demand. This research work aims to externalize the national situation of ecosystems of innovation, generation of knowledge and commercialization of the same for its use in the post of the development and consolidation of the HUB project. In this article, an exploratory research was carried out for the selection of relevant diagnostic information on these issues at the global and regional level, and a deductive study of situations already established to situations that are emerging in our country as novel fields of opportunity in difficult situations. There are disparities in the results of the HUBs at the national level, due to the difficulties that this project has generated in trying to adapt to their local realities, despite this, they have been able to generate operating regulations that support the efforts of this project and also the status regarding the activities in which each one is.

Keywords: innovation, entrepreneurship, technology transfer, HUBS

\section{Resumen}

Este artículo permite exponer el fomento a la innovación, el emprendimiento y la transferencia de tecnología y la importancia de una sociedad demandante de cosas nuevas, producto de ello se genera una necesidad de satisfacción cada vez más exigente de quienes las consumen y hacen que la generación de nuevos productos, bienes o servicios sean mayormente demandados. Este trabajo de investigación pretende exteriorizar la situación nacional de ecosistemas de innovación, generación de conocimiento y comercialización del mismo para su aprovechamiento en post del desarrollo y consolidación del proyecto HUB. En este artículo se realizó una investigación exploratoria para la selección de información relevante del diagnóstico de éstas temáticas a nivel mundial y regional, y un estudio deductivo de situaciones ya establecidas a situaciones que están surgiendo en nuestro país como campos novedosos de oportunidad en situaciones difíciles. Existen disparidad de resultados de los HUB's a nivel 
nacional, debido a las dificultades que este proyecto ha generado al tratar de adaptarse a sus realidades locales, a pesar de ello, se han logrado generar una normativa de funcionamiento que de soporte a las gestiones de este proyecto y también el estado respecto de las actividades en las que se encuentran cada uno.

Palabras Clave: innovación, emprendimiento, transferencia de tecnología, HUBS.

\section{Introducción}

Como bien sabemos la innovación ha estado asociado tradicionalmente a la evolución, a utilizar el conocimiento, y generarlo si es necesario, para crear bienes, productos, servicios, procesos o tecnología, que son nuevos para una determinada organización, o mejorar los ya existentes. Entonces la innovación se conoce como un cambio o transformación de algo ya conocido a algo novedoso u original, cuando hablamos de innovación automáticamente se lo relaciona a un contexto empresarial y tecnológico, sobre todo si el producto, bien o servicio que se ha introducido en un mercado tiene éxito sostenido que permite obtener beneficios significativos de este. El emprendimiento en la literatura común lo conocemos como crear o gestionar un negocio propio el cual permite la generación de espacios de trabajo no solo para quien lo gestiona sino también para personas que no cuentan con uno, así pues, el emprendimiento está vinculado a iniciativas de supervivencia producto del desempleo o de situaciones complicadas de localidades que presentan considerables índices de desocupación o por una inspiración de una idea de negocio viable y novedosa. La transferencia de tecnología se genera a partir de nuevas iniciativas y negocios que generan conocimiento y están establecidas en un conjunto de acciones orientadas a proporcionar una ventaja comercial en el mercado respecto de su competencia, puesto que se refiere a las capacidades investigadoras y los resultados de las actividades de I+D que efectúan sus centros o ares de investigación especializados, así como los centros de educación superior, centros de innovación, entre otros. EI HUB Centro es un espacio en red con 8 instituciones de educación superior y la Corporación Ecuatoriana para el Desarrollo de la Investigación y la Academia que forman parte, es decir, son miembros de la red. Esta organización (red) promueve un ecosistema innovador, de emprendimiento y de asistencia especializada en transferencia de tecnología, innovación, emprendimiento y además capacitación para negocios que emergen al mercado y que requieren de una guía especializada para la consolidación de su negocio. 


\section{Metodología}

El tema que se va a desarrollar es el fomento a la innovación, emprendimiento y transferencia de tecnología a través del proyecto hub centro impulsado por la secretaría nacional de educación superior por esta razón se utilizará como metodología el estudio exploratorio ya que es un tema que no se ha estudiado nunca antes en el Ecuador, por ello se tomará como ejemplos a los casos de éxito a nivel mundial y latinoamericano para que se adapte a la realidad del país y su zona específica.

Así pues, se manejará el método lógico deductivo para aplicar casos generales a nuestro caso específico a nivel del estado, ya que la Secretaría de Educación Superior Ciencia, Tecnología e Innovación - SENESCYT creó los HUBS a nivel nacional centralizándolos por zonas mediante reglamentos que permiten su funcionamiento legal y su sostenibilidad.

\section{Desarrollo y Discusión}

\subsection{Innovación}

Para comenzar la innovación lleva décadas presente en nuestra sociedad ya que es considerada como un conjunto de actividades inscritas en un determinado periodo de tiempo y lugar que conducen a la introducción con éxito en el mercado, por primera vez, de una idea en forma de nuevos o mejores productos, servicios o técnicas de gestión y organización [1].

Como sabemos la innovación está ligada al emprendimiento y a la transferencia de tecnología por esto Peter Drucker [2] destaca que:

La innovación es la función específica del emprendimiento, ya sea en una empresa existente, en una institución de servicio público o en un nuevo negocio comenzado por un individuo solitario en la cocina de su casa. Es el medio a través del cual el emprendedor crea nuevos recursos generadores de riqueza o dota a los recursos existentes de mayor potencial para crearla. [2]

De ahí que donde no exista proceso de innovación (empresas de base abierta) no se les puede dar el calificativo de empresas. De igual manera, la persona o personas que las administre o las maneje, no se les podrá llamar nunca empresarios [3].

Entonces la innovación nace a partir de las necesidades que los seres vivos requieren o de una idea que representará una futura necesidad para su confort y comodidad tal como la compañía Apple fundada por Steve Jobs en abril de 1976, por estas razones, Hernández Ascanio et al. mencionan que, 'la innovación supone el proceso por el que 
se buscan formas diversas, creativas y nuevas para satisfacer las necesidades aún no cubiertas, satisfechas de manera deficiente o emergente' [4].

A medida que las épocas avanzan este concepto también va envolviendo mayores espacios y adaptándose a las nuevas tendencias de la sociedad, por eso actualmente se centra en sapiencias tecnológicas en vista de que la innovación engloba un amplio conjunto de actividades dentro de las empresas, que contribuyen a generar nuevos conocimientos tecnológicos o a mejorar la utilización de los ya existentes. Estos conocimientos son aplicados a la obtención de nuevos bienes y servicios, así como nuevas formas de producción [5].

\subsection{Emprendimiento}

Historialmente el emprendimiento comenzó a finales del siglo XVII y principios del XVIII con Richard Cantillon y Adam Smith, que era fundamental para la economía clásica, desde sus inicios, entonces Orrego Correa menciona que:

El emprendimiento ha sido una actividad propia del ser humano, es parte de su esencia, no es ajeno a sí mismo; es en su ser interior donde se produce la convicción para enfrentar la acción exterior, realización que implica encarar las incertidumbres presentes en el contexto. [6]

Ahora bien como mencionan Duarte y Ruiz Tibana:

Cuando una sociedad necesita transformarse y lograr mejores condiciones de vida y cimentar propuestas incluyentes en un Estado participativo y en el reconocimiento de los derechos de los ciudadanos, es desde esta perspectiva que adquiere sentido y valor una propuesta de fomento a la Cultura del Emprendimiento, que convoque energías, y avale espacios de creación e innovación, materializados en oportunidades reales para los seres humanos como protagonistas de las propias transformaciones y el mejoramiento de sus contextos. [7]

A medida que el tiempo ha avanzado en el siglo XXI nació un emprendedor profesional, por ello Rodríguez Ramírez menciona que, 'las investigaciones de percepciones de los ejecutivos describen el emprendimiento o espíritu emprendedor con términos como innovador, flexible, dinámico, capaz de asumir riesgos, creativo y orientado al crecimiento' [8].

De modo que se puede decir que el emprendimiento ha heredado también la visión normativa de la administración, desde la cual, la mayoría de las entidades emprendedoras colocan su énfasis en recetas, llamadas en este caso 'planes de 
negocios' estructurados en diferentes estudios y en el uso de una serie de procedimientos organizacionales, que impactan las nuevas propuestas de un alto índice de funcionalidad [9].

Por consiguiente, un emprendimiento nace de una persona que tenga una iniciativa viable que tenga las condiciones para ser calificado como un proceso de emprendimiento, Guzmán Vásquez y Trujillo Dávila mencionan que, 'conlleva un balance de comportamiento social y comportamiento económico, que, a su vez, permite la generación de valor social y valor económico' [10].

\subsection{Transferencia de tecnología}

La innovación y el emprendimiento van ligados a la transferencia de tecnología así pues la Asociación Española para la Calidad define que es, 'un proceso de transmisión científica, tecnológica, del conocimiento, de los medios y de los derechos de explotación, hacia terceras partes para la fabricación de un producto, el desarrollo de un proceso o la prestación de un servicio, contribuyendo al desarrollo de sus capacidades' (Asociación Española para la Calidad [AEC]) [11].

Por lo tanto, la transferencia de tecnología es la transmisión, en ocasiones la creación, de dicha tecnología con o sin la consiguiente transmisión simultánea de bienes y servicios. El efecto de la transferencia de tecnología puede ser revolucionario o inexistente, dependiendo de los incentivos de ambas partes en el éxito de la transferencia y de los impedimentos de la dispersión tecnológica [12].

De modo que Insulza y Magendzo menciona que:

Existe un amplio consenso sobre la importancia de la transferencia y comercialización de nuevas tecnologías para el desarrollo de los países, pero hacerlo exitosamente requiere de una serie de conocimientos y capacidades específicas que intersectan espacios de conocimiento en ciencia, tecnología y negocios.

Desde hace dos décadas se ha venido desarrollando una ciencia que formaliza y sistematiza los conocimientos sobre transferencia $y$ emprendimiento tecnológico. [13]

De ahí que la transferencia de tecnología tiene características diferentes que dependen de si ella es externa o interna, es decir, si se da entre sistemas productivos o países, o entre sectores económicos o unidades productivas de un mismo país [14].

Actualmente en el Ecuador los cimientos han provenido de la Secretaría de Educación Superior Ciencia, Tecnología e Innovación - SENESCYT y una Corporación que comparte y aplica estos conocimientos como es CEDIA ya que presenta un pacto 
por la Innovación, misma que la (Corporación Ecuatoriana para el desarrollo de la Investigación y la Academia - CEDIA) define como:

\begin{abstract}
Una iniciativa de la Coordinación de Innovación y Transferencia Tecnológica para generar alianzas estratégicas con los diferentes actores públicos y privados del ecosistema nacional de innovación, con el fin de definir campos de colaboración orientados a potenciar los procesos de innovación y transferencia de tecnología desde la academia hacia el sector productivo.
\end{abstract} [15]

\title{
3.4. El concepto de HUB ITT: Mundo, Latinoamérica y Ecuador
}

Después de analizar los conceptos de innovación, emprendimiento y transferencia de tecnología ahora podemos abordar información referente a las agencias de innovación alrededor del mundo.

Entre los diferentes índices relacionados con la situación de la innovación en el mundo, empezamos por hacer referencia al Índice Global de Innovación (GII, por sus siglas en inglés). Este índice, elaborado desde 2007 y cuya redacción lidera la escuela de negocios INSEAD, se basa en una amplia visión de la innovación, con la inclusión de indicadores que van más allá de las medidas tradicionales.

En primer lugar, a nivel Latinoamericano Ruta N, viene siendo una de las agencias más transformadoras. Entre las acciones a destacar se encuentra la alta proporción de instrumentos destinados a fomentar el desarrollo de conglomerados (clusters) específicos, algunos de ellos existentes pero la mayoría en nuevos sectores de la economía. Por otra parte, cuenta con un programa de Landing empresarial, mediante el cual busca atraer empresas de base tecnológica al ecosistema de Medellín, con la finalidad de que la ciudad se vuelva un referente en temas tecnológicos a nivel latinoamericano y mundial.

En segundo lugar, hay un grupo de agencias latinoamericanas que presentan un enfoque estratégico mixto y operan en un SNI por consolidar y con una baja diversificación productiva. Estas agencias destinan una parte de sus recursos a potenciar y fortalecer los sectores existentes, y algunos recursos a desarrollar sectores con potencial en el país. En este grupo se destacan los esfuerzos de CORFO (Corporación de Fomento de la Producción) por medio de sus programas estratégicos enfocados en alimentos saludables, minería, pesca y acuicultura, economía creativa, industrias inteligentes e industria solar, entre otros.

En el caso de FINEP (Financiadora de Estudos e Projetos), sus proyectos estratégicos se orientan a los sectores de energía, biocombustibles, tecnologías limpias, salud y bienestar, agricultura y espacio. ANII (Agencia Nacional de Investigación e Innovación) 


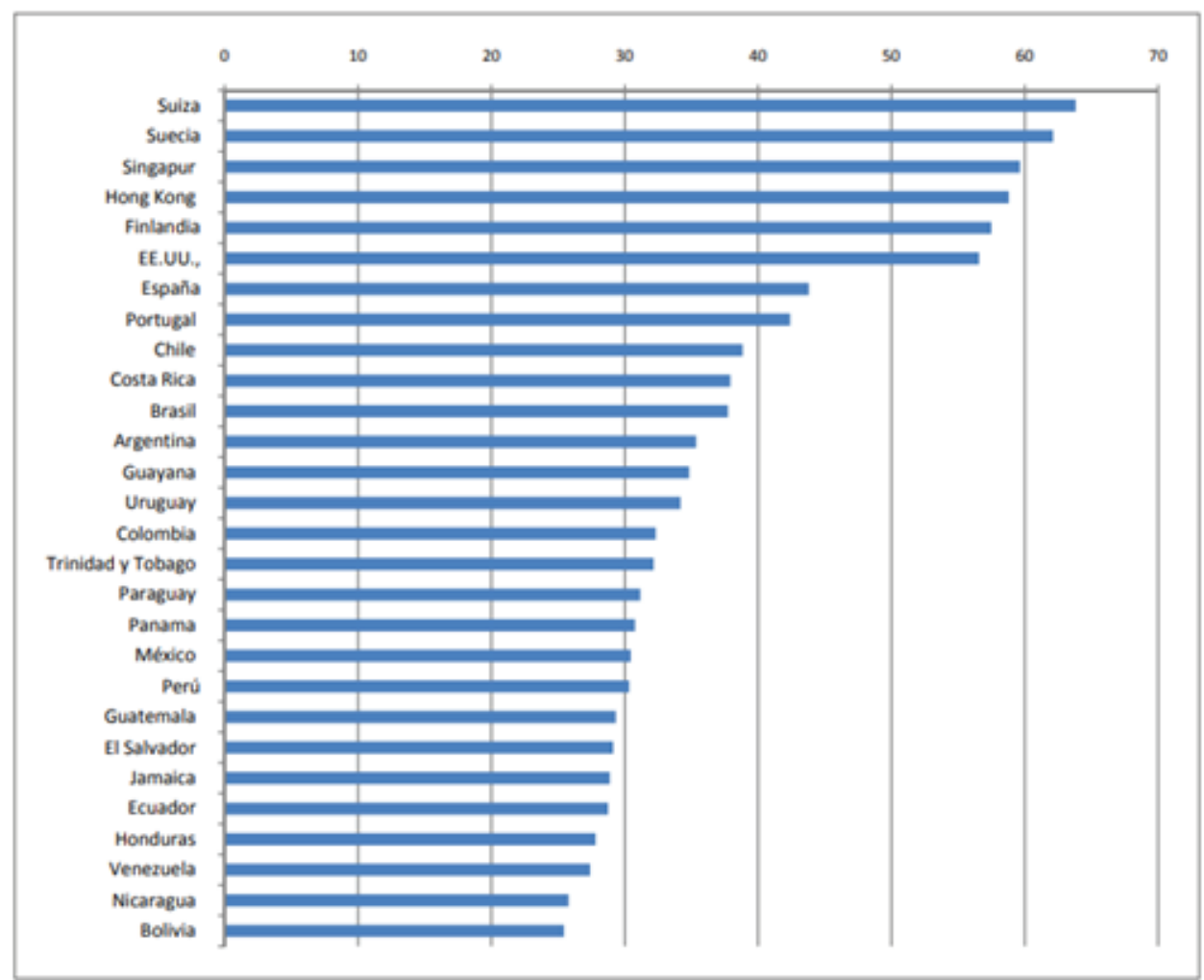

Figure 1

ALC y el Índice Global de Innovación 2011. Fuente: Referencia [16].

apunta a sectores priorizados por el Gabinete Interministerial, que son software, servicios informáticos y producción audiovisual, salud, producción agropecuaria y agroindustrial, educación y desarrollo social, logística y transporte y turismo.

Asimismo, ANCPYT (Agencia Nacional de Promoción Científica y Tecnológica) cuenta con un fondo específicamente destinado a la industria del software y con fondos sectoriales para desarrollar capacidades críticas en áreas de alto impacto potencial y transferencia permanente al sector productivo (energías renovables, agroindustria, salud y medio ambiente y cambio climático, entre otros).

El tercer grupo incluye a las agencias que se enfocan principalmente en el mejoramiento de empresas de sectores existentes. Hay dos subgrupos. Por un lado, IRAP (Industrial Research Assistance Program), CDTI (Centro para el Desarrollo Tecnológico Industrial) y SENACYT (Secretaría Nacional de Ciencia y Tecnología e Innovación), que operan en entornos con un nivel medio de diversificación, pero con diferentes niveles de desarrollo del SIN [17].

En el Ecuador tenemos un caso de éxito que es la Corporación Ecuatoriana para el desarrollo de la Investigación y la Academia - CEDIA un espacio donde potencian proyectos de innovación, emprendimiento y transferencia de tecnología, tales como: 
593 TrendLab: Es el primer laboratorio de tendencias tecnológicas en Ecuador, una muestra de que es posible juntar en un mismo espacio a estudiantes, investigadores, emprendedores, empresarios y sector público (cuádruple hélice) para analizar tendencias que desemboquen en la creación de ideas y desarrollo de productos/servicios para crear startups que permitan impulsar la innovación tan necesaria para avanzar hacia un próximo estadio de desarrollo [18].

Fondo 1 a 1 ITT: Por cada dólar que el sector privado coloque, CEDIA, en representación del sector académico, colocará su equivalente con el objetivo de resolver una necesidad o problemática de una empresa a través de un proceso de I+D+i [19].

Acorde a lo que define la (Corporación Ecuatoriana para el desarrollo de la Investigación y la Academia) las:

Brigadas de Propiedad Intelectual, son un mecanismo a través del cual, las Instituciones de Educación Superior, tienen la posibilidad de hacer una valoración neutral y técnica-legal de las creaciones e invenciones generadas dentro de sus organizaciones, ya sea por sus investigadores, o grupos de investigación y en general cualquier miembro de su organización, para tomar decisiones técnicas sobre la gestión de las mismas (protección/transferencia). [20]

En un mundo cada vez más globalizado que nos plantea nuevos retos y desafíos se requiere innovación e ingenio, es por eso que la Secretaría de Educación Superior, Ciencia, Tecnología e Innovación [21], presentó los HUB's Universitarios de Innovación y Transferencia de Tecnología, una iniciativa que busca que las ideas de nuestros jóvenes aporten al desarrollo económico del Ecuador el 26 de abril del 2018.

Los HUB's son un punto de partida para avanzar a una política de investigación que se sostenga en el tiempo, 'en donde el sector productivo, la academia y el gobierno se articulen para apoyar a los jóvenes que quieran emprender'.

Se implementará los HUB's en Quito, Guayaquil, Manabí, Cuenca, Ibarra y en la zona 3 en las provincias de Tungurahua, Cotopaxi y Chimborazo, que se ubicarán estratégicamente por zonas, con el objetivo de tener una planificación y organización nacional con visión territorial: HUB Z1- 2; HUB Z3-4; y, HUB Z6-7.

Por su parte, Lourdes de la Cruz, subsecretaria de Innovación y Transferencia Tecnológica, explicó que estos espacios en red buscan transformar las ideas de los jóvenes emprendedores en empresas que sean sostenibles y escalables.

Los innovadores tendrán acompañamiento durante todo el desarrollo de su idea, para construir un prototipo y generar un modelo de negocio que además sea de impacto internacional.

Añadió que, en Quito, ciudad en la que se presentó el primer Hub, participarán las universidades de las Fuerzas Armadas (ESPE), la Escuela Politécnica Nacional (EPN), la 
Pontificia Universidad Católica (PUCE), la Universidad Politécnica Salesiana (UPS), y la Universidad Central del Ecuador (UCE).

De esta manera, la SENESCYT confía en que los jóvenes con su ingenio e innovación puedan ser líderes innovadores, revolucionar el mundo, cambiar sus vidas, y las de miles de personas que se beneficiarán con sus creaciones.

Esta modalidad ha sido calificada como trabajo en red, iniciativa de la SENESCYT que contará con un presupuesto de 1.5 millones de dólares a nivel nacional [22].

\subsection{Constitución de la República del Ecuador}

En un estado existen leyes y reglamentos que regulan las actividades y representan los deberes y derechos de las personas que se encuentran dentro de su sociedad por esto la Constitución de la República del Ecuador que fue modificada por última vez el 01 de agosto del año 2018 entre sus artículos más relevantes para el tema a estudiar nos muestra que se debe determinar las políticas de investigación e innovación del conocimiento, desarrollo y transferencia de tecnologías, necesarias para el desarrollo regional, en el marco de la planificación nacional posteriormente generando, adaptando y difundiendo conocimientos científicos y tecnológicos que ayuden al desarrollo de la nación por esta razón el mismo estado reservará y entregará los recursos necesarios para que estos procesos sean viables y palpables.

Art. 262: Los gobiernos regionales autónomos tendrán las siguientes competencias exclusivas, sin perjuicio de las otras que determine la ley que regule el sistema nacional de competencias:

1. Planificar el desarrollo regional y formular los correspondientes planes de ordenamiento territorial, de manera articulada con la planificación nacional, provincial, cantonal y parroquial.

2. Gestionar el ordenamiento de cuencas hidrográficas y propiciar la creación de consejos de cuenca, de acuerdo con la ley.

3. Planificar, regular y controlar el tránsito y el transporte regional y el cantonal en tanto no lo asuman las municipalidades.

4. Planificar, construir y mantener el sistema vial de ámbito regional.

5. Otorgar personalidad jurídica, registrar y controlar las organizaciones sociales de carácter regional.

6. Determinar las políticas de investigación e innovación del conocimiento, desarrollo y transferencia de tecnologías, necesarias para el desarrollo regional, en el marco de la planificación nacional. 


\section{Table 1}

HUBS a nivel país.

\begin{tabular}{|c|c|c|}
\hline Hub & Integrantes & Información de contacto \\
\hline \multirow[t]{10}{*}{ Norte } & Universidad Politécnica Estatal del Carchi & $\begin{array}{l}\text { Msc. Winston Oviedo Teléfono: } \\
\text { 0997884797 Correo electrónico: } \\
\text { wgoviedo@utn.edu.ec }\end{array}$ \\
\hline & Universidad Técnica Del Norte & \\
\hline & $\begin{array}{l}\text { Universidad de Investigación de } \\
\text { Tecnología Experimental Yachay (Yachay } \\
\text { Tech) }\end{array}$ & \\
\hline & Universidad Técnica Luis Vargas Torres & \\
\hline & $\begin{array}{l}\text { Universidad Regional Autónoma de Los } \\
\text { Andes (UNIANDES) - extensión Tulcán }\end{array}$ & \\
\hline & $\begin{array}{l}\text { Pontificia Universidad Católica Del } \\
\text { Ecuador - sede Ibarra }\end{array}$ & \\
\hline & $\begin{array}{l}\text { Universidad Regional Autónoma De Los } \\
\text { Andes (UNIANDES) - extensión Ibarra }\end{array}$ & \\
\hline & $\begin{array}{l}\text { Pontificia Universidad Católica Del } \\
\text { Ecuador - sede Esmeraldas }\end{array}$ & \\
\hline & Universidad Estatal Amazónica & \\
\hline & Universidad Regional Amazónica IKIAM & \\
\hline \multirow[t]{5}{*}{ Quito } & Universidad Politécnica Salesiana & $\begin{array}{l}\text { Giovanni Herrera Teléfono: } 0984491293 \\
\text { Correo electrónico: } \\
\text { gpherrera@espe.edu.ec Paula Salazar } \\
\text { Teléfono: 0992716930 Correo } \\
\text { electrónico: psalazar@ups.edu.ec }\end{array}$ \\
\hline & Escuela Politécnica Nacional & \\
\hline & $\begin{array}{l}\text { Universidad de las Fuerzas Armadas } \\
\text { (ESPE) }\end{array}$ & \\
\hline & $\begin{array}{l}\text { Pontificia Universidad Católica del } \\
\text { Ecuador }\end{array}$ & \\
\hline & Universidad Central del Ecuador & \\
\hline \multirow[t]{7}{*}{ Centro } & Universidad Técnica de Ambato & $\begin{array}{l}\text { Ing. Patricio Arguello Teléfono: } \\
0992668250\end{array}$ \\
\hline & Universidad Técnica de Cotopaxi & \\
\hline & $\begin{array}{l}\text { Escuela Politécnica de Chimborazo } \\
\text { (ESPOCH) }\end{array}$ & $\begin{array}{l}\text { Correo electrónico: } \\
\text { carguello@espoch.edu.ec / } \\
\text { parguello67@hotmail.com }\end{array}$ \\
\hline & $\begin{array}{l}\text { Universidad Nacional de Chimborazo } \\
\text { (UNACH) }\end{array}$ & \\
\hline & $\begin{array}{l}\text { Universidad Regional Autónoma de Los } \\
\text { Andes }\end{array}$ & \\
\hline & Universidad Tecnológica Indoamérica & \\
\hline & $\begin{array}{l}\text { Universidad de das Fuerzas Armadas } \\
\text { (ESPE) - extensión Latacunga }\end{array}$ & \\
\hline
\end{tabular}

7. Fomentar las actividades productivas regionales. 
Table 1

Continued.

\begin{tabular}{|c|c|}
\hline Hub & Integrantes \\
\hline \multirow[t]{7}{*}{ Cuenca } & Universidad de Cuenca \\
\hline & Universidad de Azuay \\
\hline & $\begin{array}{l}\text { Universidad Nacional de Educación } \\
\text { (UNAE) }\end{array}$ \\
\hline & Universidad Politécnica Salesiana \\
\hline & Universidad Católica de Cuenca \\
\hline & Universidad Técnica Particular de Loja \\
\hline & Universidad Técnica de Machala \\
\hline \multirow[t]{6}{*}{ Guayaquil } & Universidad de Las Artes \\
\hline & Universidad Técnica de Babahoyo \\
\hline & Universidad Técnica Estatal de Quevedo \\
\hline & Universidad de Milagro \\
\hline & Universidad Estatal de Bolívar \\
\hline & $\begin{array}{l}\text { Universidad Católica Santiago de } \\
\text { Guayaquil }\end{array}$ \\
\hline \multirow[t]{12}{*}{ Manabí } & Universidad Técnica de Manabí \\
\hline & Universidad Laica Eloy Alfaro de Manabí \\
\hline & Universidad del Sur de Manabí \\
\hline & $\begin{array}{l}\text { Escuela Politécnica Agropecuaria Manuel } \\
\text { Félix López }\end{array}$ \\
\hline & $\begin{array}{l}\text { Pontificia Universidad Católica del } \\
\text { Ecuador - sede Manabí }\end{array}$ \\
\hline & Universidad San Gregorio de Manabí \\
\hline & $\begin{array}{l}\text { Universidad Tecnológica Equinoccial } \\
\text { Sede Santo Domingo }\end{array}$ \\
\hline & $\begin{array}{l}\text { Universidad Regional Autónoma de Los } \\
\text { Andes - sede Santo Domingo }\end{array}$ \\
\hline & Instituto Tecnológico Paulo Emilio \\
\hline & $\begin{array}{l}\text { Instituto Tecnológico Luis Arboleda } \\
\text { Martínez }\end{array}$ \\
\hline & Instituto Tecnológico Tsáchila \\
\hline & Instituto Tecnológico Calazacón \\
\hline
\end{tabular}

\section{Información de contacto}

Dr. Juan Pablo Suárez Chacón Teléfono:

(07) 3701444 ext: 2308 Correo

electrónico: jpsuarez@utpl.edu.ec

Daniel Merchán Teléfono: 0994492044

Correo electrónico:

Daniel.merchan@uartes.edu.ec Sabrina Ibarra Teléfono: 0996773640 Correo electrónico: Sabrina.ibarra@uartes.edu.ec

Priscila Feijó Teléfono: 0981375077

Correo electrónico:

priscilafeijocuenca@gmail.com

Fuente: Referencia [23].

8. Fomentar la seguridad alimentaria regional. 
9. Gestionar la cooperación internacional para el cumplimiento de sus competencias.

Art. 350: El sistema de educación superior tiene como finalidad la formación académica y profesional con visión científica y humanista; la investigación científica y tecnológica; la innovación, promoción, desarrollo y difusión de los saberes y las culturas; la construcción de soluciones para los problemas del país, en relación con los objetivos del régimen de desarrollo.

Art. 385: El sistema nacional de ciencia, tecnología, innovación y saberes ancestrales, en el marco del respeto al ambiente, la naturaleza, la vida, las culturas y la soberanía, tendrá como finalidad:

1. Generar, adaptar y difundir conocimientos científicos y tecnológicos.

2. Recuperar, fortalecer y potenciar los saberes ancestrales.

3. Desarrollar tecnologías e innovaciones que impulsen la producción nacional, eleven la eficiencia y productividad, mejoren la calidad de vida y contribuyan a la realización del buen vivir.

Art. 388: El Estado destinará los recursos necesarios para la investigación científica, el desarrollo tecnológico, la innovación, la formación científica, la recuperación y desarrollo de saberes ancestrales y la difusión del conocimiento. Un porcentaje de estos recursos se destinará a financiar proyectos mediante fondos concursables. Las organizaciones que reciban fondos públicos estarán sujetas a la rendición de cuentas y al control estatal respectivo [24].

\subsection{Ley Orgánica de Educación Superior - LOES}

Otra ley que ampara a los HUBS es la Ley Orgánica de Educación Superior ya que los HUBS están conformados principalmente por instituciones de educación superior e instituciones públicas o privadas externas que cumplan con los requisitos para adherirse como miembros según se presenta en los convenios y acuerdos pertinentes emitidos por la Secretaría de Educación Superior Ciencia, Tecnología e Innovación - SENESCYT. Por lo tanto, la Educación Superior es la encargada de generar o producir conocimientos científicos a través de los estudiantes y docentes investigadores que son la razón de ser de las universidades y escuelas superiores del país

Art. 8: Serán Fines de la Educación Superior. La educación superior tendrá los siguientes fines:

1. Aportar al desarrollo del pensamiento universal, al despliegue de la producción científica y a la promoción de las transferencias e innovaciones tecnológicas; 
2. Fortalecer en las y los estudiantes un espíritu reflexivo orientado al logro de la autonomía personal, en un marco de libertad de pensamiento y de pluralismo ideológico;

3. Contribuir al conocimiento. preservación y enriquecimiento de los saberes ancestrales y de la cultura nacional;

4. Formar académicos y profesionales responsables, con conciencia ética y solidaria, capaces de contribuir al desarrollo de las instituciones de la República, a la vigencia del orden democrático, y a estimular la participación social;

5. Aportar con el cumplimiento de los objetivos del régimen de desarrollo previsto en la Constitución y en el Plan Nacional de Desarrollo;

6. Fomentar y ejecutar programas de investigación de carácter científico, tecnológico y pedagógico que coadyuven al mejoramiento y protección del ambiente y promuevan el desarrollo sustentable nacional;

7. Constituir espacios para el fortalecimiento del Estado Constitucional, soberano, independiente, unitario, intercultural, plurinacional y laico; y

8. Contribuir en el desarrollo local y nacional de manera permanente, a través del trabajo comunitario o extensión universitaria.

Art. 35: Asignación de recursos para investigación, ciencia y tecnología e innovación. Las instituciones del Sistema de Educación Superior podrán acceder adicional y preferentemente a los recursos públicos concursables de la pre asignación para investigación, ciencia, tecnología e innovación establecida en la Ley correspondiente [25]

\subsection{Código orgánico de la Economía Social de los Conocimientos, Creatividad e Innovación (Código Ingenios)}

El código Ingenios comprende reglas que ayudan a normar la incubación de ideas con potencial de mercado, estudios de mercado, producción de prototipos, desarrollo de modelos de negocio, gestión de la propiedad intelectual, redes de contactos, portafolio de inversionistas y financistas, competentes del Estado con la colaboración del sector privado, mixto, popular y solidario las instituciones de educación superior, que permitan la prestación de servicios especializados que generen valor agregado. Como se presenta en los siguientes artículos:

Art. 1: Objeto: El presente Código tiene por objeto normar el Sistema Nacional de Ciencia, Tecnología, Innovación y Saberes Ancestrales previsto en la Constitución de la República del Ecuador y su articulación principalmente con el Sistema Nacional de Educación, el Sistema de Educación Superior y el Sistema Nacional de Cultura, con la 
finalidad de establecer un marco legal en el que se estructure la economía social de los conocimientos, la creatividad y la innovación.

-Art. 4: Principios: Para la aplicación de las disposiciones contenidas en el presente Código, se observarán los siguientes principios:

1. El conocimiento constituye un bien de interés público, su acceso será libre y no tendrá más restricciones que las establecidas en este Código, la Constitución, los tratados internacionales y la Ley y, su distribución se realizará de manera justa, equitativa y democrática;

2. Los derechos intelectuales son una herramienta para la adecuada gestión de los conocimientos. La adquisición y ejercicio de los derechos de propiedad intelectual asegurarán un equilibrio entre titulares y usuarios. Además de las limitaciones y excepciones previstas en este Código, el Estado podrá adoptar las medidas necesarias para garantizar la salud, nutrición, educación, cultura, el desarrollo científico y tecnológico, la innovación y la transferencia y difusión tecnológica como sectores de importancia vital para el desarrollo socioeconómico y tecnológico del país. Nada de lo previsto en este Código podrá interpretarse de forma contraria a los principios, derechos y obligaciones establecidos en los Tratados Internacionales de los que Ecuador es parte, como parte integrante de nuestro ordenamiento jurídico;

3. La formación del talento humano es el factor primordial de una economía social basada en los conocimientos, la creatividad y la innovación, razón por la cual debe ser de excelencia y distribuida democráticamente;

4. El conocimiento se desarrollará de manera colaborativa y corresponsable;

5. La generación, transmisión, gestión, uso y aprovechamiento de los conocimientos, la creatividad, la tecnología, la innovación y los conocimientos tradicionales se orientarán hacia la realización del buen vivir, buscando la satisfacción de las necesidades de la población, el efectivo ejercicio de los derechos y el aprovechamiento biofísicamente sustentable de los recursos del país, en el marco de la garantía de la reproducción de la vida;

6. La soberanía sobre los conocimientos es objetivo estratégico del Estado para garantizar a las personas la generación, transmisión, gestión, uso y aprovechamiento de los conocimientos, tecnología y la innovación y así materializar el buen vivir;

7. La formación académica y la investigación científica deben contribuir a la realización de los objetivos del Plan Nacional de Desarrollo; 
8. La generación, transmisión, gestión, uso y aprovechamiento de los conocimientos, la tecnología, la innovación y los conocimientos tradicionales deberán primordialmente promover la cohesión e inclusión social de todos los ciudadanos;

9. Las actividades vinculadas a la economía social de los conocimientos, la creatividad y la innovación, se desarrollarán en un marco de igualdad de oportunidades, coordinación, transparencia, calidad, evaluación de resultados y rendición de cuentas;

10. En el funcionamiento de la economía social de los conocimientos, la creatividad y la innovación, se establecerán los mecanismos de descentralización y desconcentración pertinentes, que permitan una gestión eficiente y cercana al territorio;

11. La ética en la ciencia, tecnología, innovación deberá estar orientada a la satisfacción de necesidades y a la preservación de la dignidad humana y sus aplicaciones deberán ser racionales, pluralistas y justas;

12. Los procesos investigativos y generadores de tecnología e innovación, deberán precautelar y proteger los derechos, dignidad e integridad física y psicológica de las personas que intervengan en ellos. Cualquier riesgo o afectación sobre los derechos de las personas o la naturaleza, deberá ser legítima, proporcional y necesaria. En los casos pertinentes, se deberá contar con el consentimiento libre, previo e informado de los posibles afectados;

13. Se garantiza la libertad de investigación y desarrollo tecnológico en el marco de la regulación y limitaciones que, por razones de seguridad, salud, ética o de cualquier otra de interés público, determine la Ley;

14. La creatividad es consustancial a las personas y representa un elemento trascendental para la economía social de los conocimientos, la creatividad y la innovación. El Estado deberá reconocer, proteger e incentivar la creatividad como mecanismo fundamental de solución de problemas, satisfacción de necesidades de la sociedad y la realización individual en interrelación con la investigación responsable, la innovación social y los conocimientos tradicionales;

15. El Estado propiciará el entorno favorable para la expansión y fortalecimiento de las actividades artísticas y culturales, incentivando, principalmente, la libre creación; la investigación en el arte y la cultura, con enfoque de igualdad de género y no discriminación; así como, la interacción de éstas con las otras actividades de la economía social basada en los conocimientos, la creatividad y la innovación;

16. La biodiversidad y el patrimonio genético son propiedad inalienable, imprescriptible e inembargable del Estado; no pueden ser privatizado y, su acceso, uso y aprovechamiento se realizará de forma estratégica procurando la generación de los conocimientos endógenos y el desarrollo tecnológico nacional; 
17. El espacio público deberá contribuir a la generación y difusión del conocimiento, en particular tratándose de creaciones culturales y artísticas. El Estado deberá otorgar todas las facilidades para que el espacio público sea utilizado en beneficio de creadores y usuarios;

18. Se reconoce el diálogo de saberes como el proceso de generación, transmisión e intercambio de conocimientos científicos y conocimientos tradicionales, para la concreción del Estado Plurinacional e Intercultural; y

19. Por su magnitud e impacto económico, social y político, el Estado impulsará de manera prioritaria las actividades de investigación y desarrollo tecnológico en sectores económicos denominados como industrias básicas.

Art. 18: Los espacios para el desarrollo del conocimiento y de ecosistemas de innovación. Son espacios definidos territorialmente donde se concentran servicios públicos y privados necesarios para democratizar la generación, transmisión, gestión y aprovechamiento del conocimiento, en los que interactúan y cooperan los actores del Sistema, orientados a facilitar la innovación social. En estos espacios, de impacto nacional, regional o local, se estimulará y gestionará los flujos colaborativos de conocimiento y tecnología entre todos los actores de la economía social de los conocimientos, la creatividad y la innovación que impulsen el emparejamiento y la transferencia tecnológica, la generación de capacidades sociales para la creación y el crecimiento de emprendimientos innovadores de base tecnológica entre sus miembros y otros actores.

Estos espacios para el desarrollo del conocimiento y de ecosistemas de innovación, son:

1. Las zonas especiales de desarrollo económico tecnológicos;

2. Los territorios orientados a la investigación y conocimiento;

3. Los parques científicos-tecnológicos;

4. Los parques tecno-industriales;

5. Los centros de transferencia de tecnología; y

6. Otros espacios que fueran necesarios crear para la plena implementación y el logro de los fines del Sistema. El reglamento correspondiente establecerá el régimen y condiciones aplicables a cada uno de los espacios descritos en el inciso anterior.

Sin perjuicio de lo anterior, otros espacios para el desarrollo de conocimiento y de ecosistemas de innovación podrán surgir de manera espontánea, los cuales, para poder 
acceder a financiamiento de fondos estatales, deberán estar debidamente acreditados bajo las normas de este Código.

Art. 19: De las Zonas Especiales de Desarrollo Económico Tecnológicos. Se podrán constituir Zonas Especiales de Desarrollo Económico Tecnológicas ZEDES, en el marco del Código Orgánico de la Producción, Comercio e Inversiones, para ejecutar actividades científicas, de transferencia, desarrollo y manufactura tecnológica e innovación. Estos espacios se orientarán al impulso, la creación y el desarrollo de emprendimientos, transferencia de tecnología e innovación y serán normadas por la entidad rectora del Sistema en coordinación con la autoridad rectora de la política productiva.

Art. 76: Emprendimiento innovador. Es un proyecto orientado al desarrollo de un nuevo o significativamente mejorado bien o servicio cuyo factor fundamental es el uso del conocimiento que se genera a partir de procesos de investigación, desarrollo experimental y tecnológico o procesos creativos con base científica, cuyo fin último es su introducción en el mercado.

La Secretaría de Educación Superior, Ciencia, Tecnología e Innovación, en coordinación con el resto de actores de este sistema, fomentará los procesos de innovación social necesarios para impulsar emprendimientos innovadores.

Art. 78: De la incubación de emprendimientos innovadores. - Los organismos competentes del Estado, con la participación del sector privado, mixto, popular y solidario o las instituciones de educación superior, establecerán mecanismos que permitan la prestación de servicios especializados para el desarrollo de emprendimientos innovadores y su consecuente generación de valor agregado.

Estos servicios especializados consisten en actividades relacionadas con la búsqueda de ideas con potencial de mercado, estudios de mercado, producción de prototipos, desarrollo de modelos de negocio, gestión de la propiedad intelectual, redes de contactos, portafolio de inversionistas y financistas, dotación de espacios físicos compartidos, entre otros definidos por la Secretaría de Educación Superior, Ciencia, Tecnología e Innovación.

Art. 627: Puntaje adicional en procesos de contratación pública para emprendedores. Los emprendimientos que hayan nacido de un espacio de innovación acreditado y que sean proveedores del Estado recibirán puntajes adicionales en los procesos de contratación establecidos en la Ley Orgánica del Sistema Nacional de Contratación Pública [26].

\subsection{Acuerdo No. SENESCYT - $2019-121$}

El 29 de octubre de 2019 la Secretaría Nacional de Educación Superior Ciencia, Tecnología e Innovación - SENESCYT emitió el: Acuerdo No. SENESCYT - 2019 -121 que es un Reglamento para el funcionamiento de los espacios en Red, denominados 
'HUBS de Innovación y Transferencia de Tecnología, que consta de IX capítulos dentro de los cuáles se contempla:

\subsubsection{Capítulo I: Objeto, ámbito y definiciones}

En este capítulo se consideran definiciones como: aceleradoras de empresas, acompañamiento integral, CATI (Centros de Apoyo a la Tecnología y la Innovación), Centros de Transferencia de tecnología. Emprendimiento innovador, Espacio de trabajo colaborativo o coworking, Espacios y agentes de innovación, Ganancias económicas, HUBS, Incubadoras, Innovación, Innovación abierta, Laboratorio de prototipado, Nodo de operación del HUB, Oficina de gestión de propiedad intelectual, otros espacios de transferencia de tecnología, sede de operación del HUB, Sistema Nacional de Ciencia, Tecnología, Innovación y Saberes ancestrales y Transferencia de tecnología.

\subsubsection{Capítulo II: Misión, objetivos y conformación de los HUBS}

-Art. 4: Misión: El HUB es un espacio de trabajo en red, que fomenta la creatividad, innovación e iniciativas emprendedoras a través de métodos, técnicas, instrumentos y herramientas que permiten el desarrollo y encadenamiento productivo, social y transferencia de tecnología de forma articulada con los sectores público y privado, nacional e internacional bajo un esquema de mejoramiento continuo, alineados a las vocaciones productivas y de los sectores estratégicos territoriales.

Art. 5: Objetivo: El objetivo de los HUBS es el fomento de la innovación, el emprendimiento y la transferencia de tecnología desde las instituciones que lo conforman, propiciando la participación de los sectores académicos, productivos y sociales, mediante la conformación de espacios de red [27].

Los HUBS se podrán integrar por Instituciones de Educación Superior; Institutos Públicos de Investigación, Empresas Públicas y Privadas vinculadas a actividades de Ciencia, Tecnología e Innovación; Gobiernos Autónomos Descentralizados; Organizaciones sin fines de lucro; otros actores Públicos o Privados, Nacionales o Extranjeros, generadores y gestores del conocimiento.

\subsubsection{Capítulo III: Gestión y funcionamiento de los HUBS}

En este capítulo se observa las obligaciones: del Directorio, del Coordinador General, del Administrador de los recursos, de los miembros del HUB, de la Secretaría de Educación Superior Ciencia, Tecnología e Innovación y del Comité Técnico. 


\subsubsection{Capítulo IV: Estructura de los HUBS}

Los HUBS podrán ofertar los siguientes servicios especializados:

Incubación y aceleración de emprendimientos innovadores, espacios de coworking, asesoría especializada, capacitación y formación continua en innovación y transferencia de tecnología, financiamiento en actividades de innovación, emprendimiento y transferencia de tecnología, red de mentores.

\subsubsection{Capítulo V: Gestión de recursos financieros}

La Secretaría de Educación Superior Ciencia, Tecnología e Innovación destinará recursos de su presupuesto para financiar a los HUBS siempre y cuando cuente con la disponibilidad presupuestaria y certificación de fondos correspondiente de acuerdo a lo establecido en el Código Orgánico de Planificación y Finanzas Públicas.

\subsubsection{Capítulo VI: Bienes}

Art. 27: Ubicación y custodia de los bienes. Los bienes adquiridos con los recursos asignados por la Secretaría de Educación Superior Ciencia, Tecnología e Innovación se ubicarán en la sede y nodos del HUB según lo aprobado por el coordinador general [27].

\subsubsection{Capítulo VII: Gestión de propiedad intelectual}

Art. 30: Gestión de propiedad Intelectual. EL HUB deberá establecer mecanismos para la asesoría y gestión de la propiedad intelectual de los proyectos desarrollados por las instituciones que lo conforman y cuyo aprovechamiento sea canalizado a través del HUB [27].

\subsubsection{Capítulo VIII: Monitoreo y seguimiento}

Art. 31: Instancias Responsables del monitoreo y seguimiento de las actividades del HUB. El monitoreo y seguimiento de las actividades desarrolladas por el HUB será realizado por las siguientes instancias:

1. El Directorio;

2. La Coordinación General del HUB; 
3. La Secretaría de Educación Superior Ciencia, Tecnología e Innovación, a través de la Subsecretaría de Innovación y Transferencia de Tecnología o las Coordinaciones Zonales, según el caso.

Art. 32: Monitoreo y seguimiento de actividades. Las actividades planificadas por el HUB para el cumplimiento de sus objetivos, serán monitoreadas tanto en su aspecto técnico, administrativo y financiero por las instancias definidas en el artículo anterior.

Los Informes de monitoreo y seguimiento serán remitidos a la Secretaría de Educación Superior Ciencia, Tecnología e Innovación, por el Coordinador General de manera trimestral o conforme a lo establecido en cada convenio suscrito en caso de ser necesario, se podrá solicitar aclaraciones a los informes o hacer recomendaciones al mismo [27].

\subsubsection{Capítulo IX: Proceso de liquidación del convenio}

Art. 34: Terminación del convenio. La terminación del convenio se dará por cualquiera de las causales determinadas en el convenio de conformación del HUB, y se iniciará el correspondiente proceso de liquidación [27].

\subsection{Ley Orgánica de emprendimiento e innovación}

La ley Orgánica de Emprendimiento e Innovación entró en vigencia el 07 de enero de 2020 dentro de la cual podemos destacar ligeramente los siguientes artículos que ayudan a comprender mejor este tema.

Esta ley promueve una cultura emprendedora que forme conocimientos y proyectos innovadores y de base tecnológica generando a la par fuentes de trabajo para los habitantes del país y produciendo un desarrollo a nivel zonal y de estado.

Art. 1: Objeto y ámbito. La presente Ley tiene por objeto establecer el marco normativo que incentive y fomente el emprendimiento, la innovación y el desarrollo tecnológico, promoviendo la cultura emprendedora e implementando nuevas modalidades societarias y de financiamiento para fortalecer el ecosistema emprendedor.

El ámbito de esta ley se circunscribe a todas las actividades de carácter público o privado, vinculadas con el desarrollo del emprendimiento y la innovación, en el marco de las diversas formas de economía pública, privada, mixta, popular v solidaria, cooperativista, asociativa, comunitaria y artesanal.

Art. 13: Infraestructura para centros de emprendimientos. Con la finalidad de acompañar el desarrollo de emprendimientos, el ente rector de la gestión inmobiliaria del sector público brindará apoyo y facilitará instalaciones, infraestructuras o establecimientos disponibles a su cargo, mediante la suscripción de convenios, a los Gobiernos 
Autónomos Descentralizados e instituciones del gobierno central, para ser utilizados como centros de incubación gratuita para emprendedores.

De igual forma, el ente rector de la gestión inmobiliaria del sector público podrá facilitar instalaciones, infraestructuras o establecimientos a su cargo, mediante el arrendamiento a precio preferencial para ser utilizados como centros de apoyo, desarrollo y/o aceleradoras de emprendimientos de carácter público y/o privado.

La sanción o clausura a un emprendimiento no podrá perjudicar a otros emprendimientos ubicados en el mismo establecimiento.

Art. 32: Plataformas de fondos colaborativos o crowdfunding. Las plataformas de fondos colaborativos o 'crowdfunding', conectan mediante plataformas de internet a personas públicas, privadas o de la economía popular y solidaria, denominadas promotores, que requieren capital para un determinado proyecto, con o sin ánimo de lucro, con otras personas, denominadas inversores, interesadas en aportar sus recursos para la consecución de dichos proyectos, bajo determinadas condiciones y a través de distintas categorías [28].

Finalmente analizando los casos de Innovación, emprendimiento y transferencia de tecnología a nivel mundial, latinoamericano y nacional se obtiene como resultados que la Secretaría de Educación Superior Ciencia, Tecnología e Innovación - SENESCYT creo el Acuerdo No. SENESCYT - 2019 -121 para que los HUBS puedan entrar en funcionamiento amparados también por la Ley Orgánica de Emprendimiento e Innovación que ingreso en vigencia en enero del presente año.

Siendo así los diferentes HUBS que están trabajando para el desarrollo del país son:

\section{Table 2}

Avances de los HUBS a nivel país.

\begin{tabular}{|c|c|}
\hline Hubs & Avances \\
\hline Norte & $\begin{array}{l}\text {-Presentaron planes de negocio a la empresa del Ingenio Azucarero del Norte, de } \\
\text { proyectos de briquetas y platos desechables, utilizados como materia prima de los } \\
\text { subproductos derivados de la caña de azúcar para producir materiales respetuosos con } \\
\text { el ambiente. } \\
\text {-Dicta cursos virtuales gratuitos por el MIT. }\end{array}$ \\
\hline Quito & Realizó el TestingLab Innova. \\
\hline Centro & Retos de Innovación Abierta en Riobamba, Latacunga y Ambato. \\
\hline Cuenca & $\begin{array}{l}\text { Se desarrolló un Bootcamp en junio del año pasado y se planificó la ejecución de un } \\
\text { Innovation Day en la UTPL en la que invitarán a empresarios de la localidad para agilizar } \\
\text { la dinámica con los investigadores en la presentación de propuestas a sus necesidades. }\end{array}$ \\
\hline Guayaquil & $\begin{array}{l}\text { Han trabajado desde el territorio en el modelo de gestión para su respectivo HUB y } \\
\text { retos con el sector empresarial. Marcarán una hoja de ruta para convertirá a Guayaquil } \\
\text { en un Hub de Innovación }\end{array}$ \\
\hline Manabí & $\begin{array}{l}\text { Este HUB se constituyó con el objetivo de promover el desarrollo de la innovación y la } \\
\text { transferencia tecnológica, a través de un trabajo articulado entre la academia, el sector } \\
\text { productivo y el Gobierno. }\end{array}$ \\
\hline
\end{tabular}




\section{Conclusiones}

La innovación, emprendimiento y la transferencia de tecnología son campos muy amplios a nivel mundial, pero son complementarios cuando de generar y consolidar una idea de negocio se trata, en ese contexto el país da sus primeros pasos al promover este tipo de ambientes con los HUBS como organizaciones gestoras.

La normativa que se generó para el desarrollo de las gestiones del proyecto HUB fue oportuna y cumplió con las expectativas previstas tanto en sostenibilidad como en la generación de alianzas que permitan sumar esfuerzos para el cumplimiento de los objetivos.

Finalmente, los HUB's a nivel mundial se encuentran en fases diferentes debido a las situaciones locales, por ello se entiende que el fomento a ecosistemas de innovación está en un estado próspero y encaminado hacia el cambio de una cultura de conocimiento, porque cada HUB genera espacios que promueven un cambio de mentalidad hacia el progreso.

\section{References}

[1] Cilleruelo E. Google académico. 2007. Recuperado de: https://ww.revistadyo.org/ index.php/dyo/article/view/20/20

[2] Drucker P. Google académico. 2004 August. Recuperado de: http://www.sela.org/ media/3212238/r-la-disciplina-de-la-innovacion.pdf

[3] Suárez MO. Universidad tecnológica de pereira. 2004 August. Recuperado de: file: ///C:/Users/Vanesa/Desktop/HUB/papers/caja\{\%\}20de\{\%\}2Oinformaci\{\%\}C3\{\%\}B3n/ 7255-5325-1-PB.pdf

[4] Ascanio JH, Valencia TP, Montes AA. Google académico. 2016 December. Recuperado de: https://www.redalyc.org/pdf/174/17449696006.pdf

[5] Mathison L, Gándara J, Primera C, García L. Google Académico. 2007 July Recuperado de: http://ojs.revistanegotium.org.ve/index.php/negotium/article/view/ $45 / 37$

[6] Correa CIO. Redayc.org. Pensamiento \& Gestión. 2009 December. Recuperado de: https://www.redalyc.org/pdf/646/64612782008.pdf

[7] Duarte T, Tibana MR. (diciembre de 2009). Scientia Et Technica. 2009 December. Recuperado de: https://www.redalyc.org/pdf/849/84917310058.pdf

[8] Ramírez AR. Scielo. 2009 June Recuperado de: http://www.scielo.org.co/scielo.php? pid=S1657-62762009000100005\&script=sci_arttext\&tIng=pt

[9] Orrego Cl. Revista Ciencias Estratégicas. 2008. Recuperado de: file:///C: /Users/Vanesa/Desktop/HUB/papers/caja\{\%\}20de\{\%\}2Oinformaci\{\%\}C3\{\%\}B3n/

Dialnet-LaDimensionHumanaDeIEmprendimiento-2991259.pdf 
[10] Vásquez GA, Dávila TMA. Redalyc.org. 2008. Recuperado de: https://www.redalyc. org/pdf/212/21211518005.pdf

[11] Asociación Española para la Calidad (AEC). QAEC. 2019. Recuperado de: https: //www.aec.es/web/guest/centro-conocimiento/transferencia-de-tecnologia

[12] Echarri A, Pendás Á. La transferencia de tecnología. Aplicación práctica y jurídica. Recuperado de https://books.google.es/books?hl=es\&lr=\&id= g9k3qvOMYOEC\&oi=fnd\&pg=PA69\&dq=transferencia+de+tecnologia\&ots= DYizThVOyX\&sig=mYp6m63YfsGW7GJQedgzApjbfvk\#v=onepage\&q= transferencia\{\%\}20de\{\%\}20tecnologia\&f=false

[13] Insulza D, Magendzo A. Banco interamericano de desarrollo. 2016 March 3. Recuperado de: https://blogs.iadb.org/innovacion/es/ transferencia-tecnologica-no-es-fisica-cuantica/

[14] García TH. Universidad de Antioquía. Recuperado de: http://bibliotecadigital.udea. edu.co/bitstream/10495/6002/1/TapiasHeberto_1996_TransferenciaTecnologia.pdf

[15] Corporación Ecuatoriana para el desarrollo de la Investigación y la Academia CEDIA. (s.f.). CEDIA. Recuperado de: https://www.cedia.edu.ec/es/sobre-nosotros/ innovacion-y-transferencia-tecnologica/pacto-por-la-innovacion

[16] Botella C, Suárez I. Fundación Carolina - CeALCI. 2012 May. Recuperado de: file:///C:/Users/Vanesa/Desktop/HUB/papers/INNOVACION\%2OPARA\%20EL\% 20DESARROLLO\%20EN\%20AL.pdf

[17] Angelelli P, Luna F, Suaznábar C. Banco Interamericano de desarrollo. 2017 October. Recuperado de file:///C:/Users/Vanesa/Desktop/HUB/ papers/Agencias-latinoamericanas-de-fomento-de-la-innovaci\%C3\% B3n-y-el-emprendimiento-Caracter\%C3\%ADsticas-y-retos-futuros.pdf

[18] Corporación Ecuatoriana para el desarrollo de la Investigación y la Academia. (s.f.). CEDIA. Recuperado de: https://www.cedia.edu.ec/es/sobre-nosotros/ innovacion-y-transferencia-tecnologica/fondo-1-1

[19] Corporación Ecuatoriana para el desarrollo de la Investigación y la Academia. CEDIA. Recuperado de: Corporación Ecuatoriana para el desarrollo de la Investigación y la Academia

[20] Corporación Ecuatoriana para el desarrollo de la Investigación y la Academia. CEDIA. Recuperado de https://www.cedia.edu.ec/es/sobre-nosotros/ innovacion-y-transferencia-tecnologica/brigadas-de-propiedad-intelectual

[21] Secretaría de Educación Superior, Ciencia Tecnología e Innovación. 2018 September 12. Recuperado de https://www.educacionsuperior.gob.ec/ zona-1-y-2-inaugura-hub-para-la-innovacion-y-emprendimiento/

[22] Secretaría de Educación Superior, Ciencia, Tecnología e Innovación. 2018 April 26. Recuperado de https://www.educacionsuperior.gob.ec/ senescyt-presenta-los-hubs-una-red-que-fomenta-la-innovacion-y-el-emprendimiento/ 
[23] Secretaría de Educación Superior, Ciencia, Tecnología e Innovación. (s.f.). Recuperado el 29 de enero de 2020, de https://www.educacionsuperior.gob.ec/ programas-y-proyectos/

[24] Asamblea Nacional de Ecuador. Secretaría General de Comunicación de la Presidencia. 2015 December 21. Recuperado de https://www.comunicacion.gob. ec/wp-content/uploads/downloads/2016/06/CONSTITUCION-CRE-con-enmiendas. pdf

[25] Asamblea Nacional del Ecuador. Secretaría de Educación Superior, Ciencia, Tecnología e Innovación. 2010 August 4. Recuperado de https://www. educacionsuperior.gob.ec/wp-content/uploads/downloads/2012/07/loes.pdf

[26] Asamblea Nacional del Ecuador. 2016 December 9. Retrieved from: https://www. wipo.int/edocs/lexdocs/laws/es/ec/ec075es.pdf

[27] Secretaría de Educación Superior Ciencia, Tecnología e Innovación. Acuerdo No. SENESCYT -2019-121. 2019 October 29. Recuperado de: https://www. educacionsuperior.gob.ec/

[28] Asamblea Nacional del Ecuador. 20 January 7. Recuperado de: $\quad$ https:/www.asambleanacional.gob.ec/es/multimedios-legislativos/ 60908-proyecto-de-ley-organica-de 